\title{
Fine 2014: contributi economici per la dialisi domiciliare nel Lazio
}

\author{
Gianna d'Adamo \\ Nefrologa, Roma
}

Late 2014: economic contributions for home dialysis in Lazio

The Decree of the Commissario ad Acta of the Lazio Region of 12.22.2014 updates the reimbursement policies of the travel expenses for those patients that undergo hemodialysis at a hospital or centre. The Decree also contains some slight adjustments of the financial contributions for those patients on home dialysis.

We look forward to the enactment of a regional law on all aspects of home dialysis.

Keywords: Peritoneal dialysis, Home dialysis

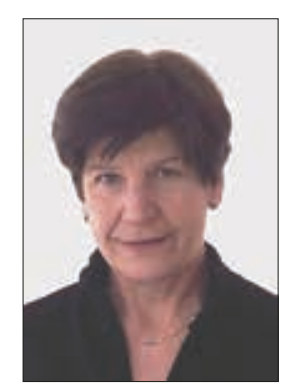

Gianna d'Adamo

\section{Lazio: Decreto del Commissario ad Acta del 22/12/2014 n. U00441}

Sul Bollettino Ufficiale della Regione Lazio n. 103 del 29/12/2014 è stato pubblicato il decreto commissariale 441 del 22/12/2014 che reca "Disposizioni normative in materia di nefropatie e dialisi e di contributi per spese di trasporto e prestazioni dialitiche e contestuale revoca della DGR n. 1614/2001".

La parte preponderante del documento è dedicata alla revisione e all'aggiornamento del sistema tariffario relativo alle spese di trasporto per le persone che effettuano emodialisi in centro, ma c'è anche una parte che riguarda la dialisi domiciliare.

Il decreto esplicita la volontà di incentivare lo sviluppo della dialisi domiciliare e, a tal fine, aggiorna i contributi a favore delle persone che scelgono i trattamenti domiciliari:

- I'ASL che ha in carico il paziente sostiene l'intero costo per impianti ed eventuali opere murarie, concordate con il responsabile dell'UO di Nefrologia e Dialisi che effettua la terapia, e fornisce le apparecchiature necessarie, tanto per l'emodialisi quanto per la peritoneale;

- il contributo annuo erogato dall'ASL per il 2014 per le

Accepted: February 10, 2015

Published online: March 5, 2015

Indirizzo per la corrispondenza:

Dr. ssa Gianna d'Adamo

Nefrologa, Roma

giannadadamo@gmail.com spese sostenute dal paziente è pari a $€ 970.46$ per l'emodialisi, a $€ 646.90$ per l'APD e a $€ 485.00$ per la CAPD. Nei prossimi anni il contributo verrà rivalutato secondo il tasso di inflazione programmato rilevabile dal DPEF. In casi eccezionali, l'ASL anticipa le spese necessarie per il trattamento domiciliare ai pazienti che versano in documentate difficoltà economiche.

È altresì confermato che:

- I'ASL o I'Azienda Ospedaliera che eroga la dialisi domiciliare devono provvedere alla consegna periodica a casa del paziente di tutti i materiali necessari, al ritiro e allo smaltimento dei rifiuti speciali prodotti dalle sedute di dialisi e alla manutenzione e/o alla sostituzione delle apparecchiature;

- in caso di malattia o di impossibilità temporanea del partner dialitico, il centro di cura deve garantire il rientro del paziente. Rientro al centro di cura o invio a domicilio di un infermiere, anche per consentire al partner un periodo di riposo.

Non è molto, ma forse è un segnale di interesse nei confronti della dialisi peritoneale che non ha ancora una normativa regionale, anche se i dati del Registro Regionale Dialisi e Trapianto Lazio mostrano un progressivo incremento dell'utilizzo della dialisi peritoneale dal 1999 al 2012:

“L'offerta di dialisi peritoneale tra i prevalenti nel 2012 è pari al 7.8\%; considerando solo i centri pubblici, l'offerta di dialisi peritoneale è del $13.4 \%$. Tra i casi incidenti, l'offerta è maggiore, pari al $12.0 \%$ e al $22.0 \%$ (centri pubblici)".

\section{Disclosures}

Financial support: No financial support was received for this submission. Conflict of interest: The author has no conflict of interest. 